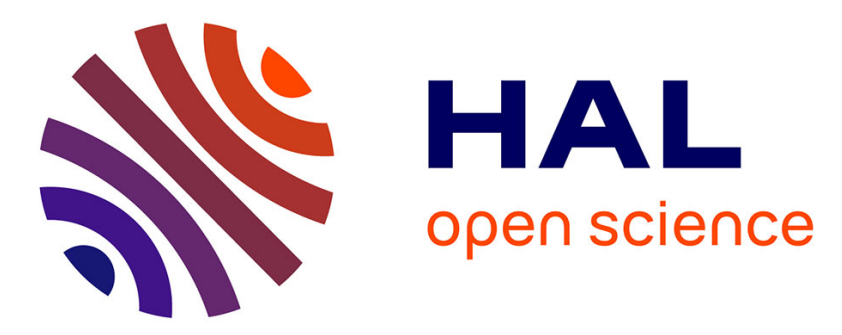

\title{
Stability analysis of singularly perturbed switched and impulsive linear systems
}

Jihene Ben Rejeb, Irinel-Constantin Morarescu, Antoine Girard, Jamal

Daafouz

\section{- To cite this version:}

Jihene Ben Rejeb, Irinel-Constantin Morarescu, Antoine Girard, Jamal Daafouz. Stability analysis of singularly perturbed switched and impulsive linear systems. 55th IEEE Conference on Decision and Control, CDC 2016, Dec 2016, Las Vegas, NV, United States. 10.1109/cdc.2016.7799117 . hal01379136

\section{HAL Id: hal-01379136 \\ https://hal.science/hal-01379136}

Submitted on 11 Oct 2016

HAL is a multi-disciplinary open access archive for the deposit and dissemination of scientific research documents, whether they are published or not. The documents may come from teaching and research institutions in France or abroad, or from public or private research centers.
L'archive ouverte pluridisciplinaire HAL, est destinée au dépôt et à la diffusion de documents scientifiques de niveau recherche, publiés ou non, émanant des établissements d'enseignement et de recherche français ou étrangers, des laboratoires publics ou privés. 


\title{
Stability analysis of singularly perturbed switched and impulsive linear systems
}

\author{
Jihene Ben Rejeb, Irinel-Constantin Morărescu, Antoine Girard and Jamal Daafouz
}

\begin{abstract}
This paper proposes a new methodology for stability analysis of singularly perturbed linear systems whose dynamics is affected by switches and state jumps. The overall problem is formulated in the framework of hybrid singularly perturbed systems and we use Lyapunov-based techniques to investigate its stability. We emphasize that, beside the stability of slow and fast dynamics, we need a dwell-time condition to guarantee the overall singularly perturbed system is globally asymptotically stable. Furthermore, we characterize this dwell-time as the sum of one term related to the stabilization of systems evolving on one time-scale (slow dynamics) and one term of the order of the parameter defining the ratio between the time-scales. As highlighted in the paper the second term is required to compensate the effect of the jumps introduced in the state of the boundary layer system by the switches and impulses affecting the overall dynamics. Some numerical examples illustrates our results.
\end{abstract}

Index Terms-Singular Perturbation, Switched systems, Reset systems, Dwell-time.

\section{INTRODUCTION}

Systems characterized by dynamical processes that evolve on different time scales are encountered in a broad domain of science going from biology [1], [2] to engineering [3], [4]. When several orders of magnitude differentiate the various time scales, the standard analysis becomes more difficult and singular perturbation theory [5], [6] is used to approximate the dynamics by decoupling the slow dynamical processes of the faster ones. The stability analysis is done separately for each time scale and under appropriate assumptions one can conclude on the stability of the overall system. Significant results related to stability analysis and approximation of solutions of singularly perturbed systems can be found in [7], [8], [9]. Various biological singularly perturbed systems are analyzed from a geometric perspective in [10].

Another feature that characterizes many physical systems is the presence of discrete events that occur during the continuous dynamics. These events may be represented either by some change of dynamics without state jumps or by impulses on the state of the system. The first type of events

\footnotetext{
This work was funded by the ANR project COMPACS - "Computation Aware Control Systems", ANR-13-BS03-004.

J. Ben Rejeb, I.-C. Morărescu, J. Daafouz are with Université de Lorraine, CRAN, UMR 7039 and CNRS, CRAN, UMR 7039, 2 Avenue de la Forêt de Haye, Vandœuvre-lès-Nancy, France. (jihene.ben-rejeb, constantin.morarescu, jamal.daafouz) (aniv-lorraine.fr

Antoine Girard, Laboratoire des signaux et systèmes (L2S), CNRS, CentraleSupélec, Université Paris-Sud, Université ParisSaclay, 3, rue Joliot-Curie, 91192 Gif-sur-Yvette, cedex, France, Antoine.Girardal2s.centralesupelec.fr
}

leads to the class of switched systems. Stabilization and exponential stability of singularly perturbed linear switched systems are considered in [11], [12]. The state jumps/resets can be physical as in the case of mechanical systems with impacts or electrical circuits with diodes, but they can also be generated by the control strategy as in the case of eventtriggering control [13]. More general hybrid systems include discrete events that can involve both switches and state jumps. This framework can be motivated by the implementation of hybrid feedback control algorithms through fast actuators. A stability result for this class of systems can be found in [4].

In this paper we focus on the stability analysis of linear switched systems affected by state jumps/resets. The main contributions of the current work are:

- a new methodology for stability analysis of linear singularly perturbed systems with switches and impulsions.

- the characterization of the minimal dwell-time between two events that ensures the stability of the overall singularly perturbed system.

It is noteworthy that, the required dwell time is the sum of two terms. The first one is the regular dwell-time guaranteeing the stability of linear switched systems evolving on one time-scale but whose dynamics do not share a common Lyapunov function. The second one is of order of the parameter characterizing the ratio between the time-scales of the processes involved in the singularly perturbed system. Our approach is based on classical singular perturbation theory [5] with Lyapunov function arguments for hybrid systems (see [14] for details).

The paper is organized as follows. Section II is devoted to preliminary results concerning singularly perturbed systems. Section III describes the switched impulsive system model in the singular perturbation form. Section IV states the main results along with its Lyapunov-based proofs. Three examples are given in Section V to illustrate the applicability of the proposed approach. Some conclusions are presented at the end of the paper.

\section{NOTATION}

Throughout this paper, $\mathbb{R}_{+}, \mathbb{R}^{n}$ and $\mathbb{R}^{n \times m}$ denote respectively, the set of nonnegative real numbers, the $n$ dimensional Euclidean space and the set of all $n \times m$ real matrices. The identity matrix of dimension $n$ is denoted by $\mathbf{I}_{n}$. We also denote by $\mathbf{0}_{n, m} \in \mathbb{R}^{n \times m}$ the matrix whose components are 
all 0. For a matrix $A \in \mathbb{R}^{n \times n},\|A\|$ denotes the spectral norm i.e. induced 2 norm. $A>\mathbf{0}(A<\mathbf{0})$ means that $A$ is positive definite (negative definite). We write $A^{\top}$ and $A^{-1}$ to respectively denote the transpose and the inverse of $A$. The matrix $A$ is said to be Hurwitz if all its eigenvalues have negative real parts. $A$ is said to be Schur if all its eigenvalues have modulus smaller than one. We also use $x\left(t_{k}^{+}\right)=\lim _{\delta \rightarrow 0, \delta>0} x\left(t_{k}+\delta\right)$.

\section{PRELIMinaries}

In this section we reformulate some results on the Lyapunov stability of linear singularly perturbed systems. These will be used in the next sections to prove the main results of the paper concerning the stability of multiple time-scale systems in presence of switches and impulses.

Let us consider the singularly perturbed linear system :

$$
\left\{\begin{aligned}
\dot{x}(t) & =A_{11} x(t)+A_{12} z(t) \\
\varepsilon \dot{z}(t) & =A_{21} x(t)+A_{22} z(t)
\end{aligned}\right.
$$

where $x(t) \in \mathbb{R}^{n_{x}}, z(t) \in \mathbb{R}^{n_{z}}$ and $\varepsilon>0$ is a small parameter. Let us assume that $A_{22}$ is non-singular and proceed with the change of variable

$$
\left(\begin{array}{l}
x(t) \\
y(t)
\end{array}\right)=\left(\begin{array}{cc}
\mathbf{I}_{n_{x}} & \mathbf{0}_{n_{x}, n_{z}} \\
A_{22}^{-1} A_{21} & \mathbf{I}_{n_{z}}
\end{array}\right)\left(\begin{array}{c}
x(t) \\
z(t)
\end{array}\right) .
$$

In the variables $x, y$ the system becomes:

$$
\left\{\begin{array}{l}
\dot{x}(t)=A_{0} x(t)+B_{1} y(t) \\
\varepsilon \dot{y}(t)=A_{22} y(t)+\varepsilon\left(B_{2} x(t)+B_{3} y(t)\right)
\end{array}\right.
$$

where

$$
\begin{aligned}
& A_{0}=A_{11}-A_{12} A_{22}^{-1} A_{21}, B_{1}=A_{12}, \\
& B_{2}=A_{22}^{-1} A_{21} A_{0}, B_{3}=A_{22}^{-1} A_{21} A_{12} .
\end{aligned}
$$

Let us make the following assumption:

Assumption 1: $A_{0}$ and $A_{22}$ are Hurwitz.

Under the previous assumption, there exist symmetric positive definite matrices $Q_{s} \geq \mathbf{I}_{n_{x}}, Q_{f} \geq \mathbf{I}_{n_{z}}$ and a positive number $\lambda$ such that:

$$
\begin{aligned}
A_{0}^{\top} Q_{s}+Q_{s} A_{0}^{\top} & \leq-2 \lambda Q_{s} \\
A_{22}^{\top} Q_{f}+Q_{f} A_{22}^{\top} & \leq-2 \lambda Q_{f}
\end{aligned}
$$

Due to space-limitations the following 3 results are given without proofs.

Proposition 1: If $A_{0}$ and $A_{22}$ are Hurwitz then there exists $\varepsilon_{1}>0$ such that

$$
V(x, y)=x^{\top} Q_{s} x+y^{\top} Q_{f} y
$$

is a Lyapunov function for system (3) for all $\varepsilon \in\left(0, \varepsilon_{1}\right)$.

In the following, let us denote $W_{s}(t)=\sqrt{x(t)^{\top} Q_{s} x(t)}$ and $W_{f}(t)=\sqrt{y(t)^{\top} Q_{f} y(t)}$. Let us also introduce the following notation $b_{1}=\left\|Q_{s} B_{1}\right\|, \quad b_{2}=\left\|Q_{f} B_{2}\right\|$ and $b_{3}=\left\|Q_{f} B_{3}\right\|$
Proposition 2: Let $\varepsilon_{1}$ as in Proposition 1, then for all $\varepsilon \in$ $\left(0, \varepsilon_{1}\right), \theta>0$ and $t \geq 0$

$$
W_{f}(t) \leq W_{f}(0) \mathrm{e}^{-\frac{\lambda}{\varepsilon} t}+\varepsilon \beta_{1} \sqrt{V(0)} \mathrm{e}^{-\lambda(1-\theta) t}
$$

where $\beta_{1}=\frac{\max \left(b_{2}, b_{3}\right)}{\lambda\left(1-\varepsilon_{1}(1-\theta)\right)}$.

Proposition 3: Let $\varepsilon_{1}$ as in Proposition 1, let $0<\varepsilon_{2}<$ $\min \left(\varepsilon_{1}, 1\right)$ then for all $\varepsilon \in\left(0, \varepsilon_{2}\right), \theta>0$ and $t \geq 0$

$W_{s}(t) \leq W_{s}(0) \mathrm{e}^{-\lambda t}+\varepsilon \beta_{2} W_{f}(0) \mathrm{e}^{-\lambda t}+\varepsilon \beta_{3} \sqrt{V(0)} \mathrm{e}^{-\lambda(1-\theta) t}$ where $\beta_{2}=\frac{b_{1}}{\lambda\left(1-\varepsilon_{2}\right)}$ and $\beta_{3}=\frac{b_{1} \beta_{1}}{\lambda \theta}$.

\section{PROBLEM FORMULATION}

In this paper, we consider a switched system of the form:

$$
\left(\begin{array}{c}
\dot{x}(t) \\
\varepsilon \dot{z}(t)
\end{array}\right)=A^{\sigma_{k}}\left(\begin{array}{c}
x(t) \\
z(t)
\end{array}\right), \forall t \in\left(t_{k}, t_{k+1}\right], k \in \mathbb{N}
$$

with impulsive dynamics:

$$
\left(\begin{array}{c}
x\left(t_{k}^{+}\right) \\
z\left(t_{k}^{+}\right)
\end{array}\right)=J^{\nu_{k}}\left(\begin{array}{c}
x\left(t_{k}\right) \\
z\left(t_{k}\right)
\end{array}\right), \quad \forall k \geq 1
$$

where $x(t) \in \mathbb{R}^{n_{x}}, z(t) \in \mathbb{R}^{n_{z}}, 0=t_{0}<t_{1}<\ldots$ are the instants of discrete events (switches, impulses or both), $\sigma_{k} \in \mathcal{I}$ and $\nu_{k} \in \mathcal{J}$ with $\mathcal{I}$ and $\mathcal{J}$ finite sets of indices, $A^{i}$ and $J^{j}$ are matrices of appropriate dimensions for all $i \in \mathcal{I}$, $j \in \mathcal{J}$, and $\varepsilon>0$ is a small parameter characterizing the time scale separation between the slow dynamics of $x$ and the fast dynamics of $z$.

Remark 1: Switches and impulses can, but need not, be concomitant. Indeed, if $\sigma_{k}=\sigma_{k+1}$ and $J^{\nu_{k}} \neq \mathbf{I}_{n_{x}+n_{z}}$, then at time $t_{k}$ an impulse occurs but no switch. Similarly, if $\mathbf{I}_{n_{x}+n_{z}} \in\left\{J^{j} \mid j \in \mathcal{J}\right\}$, for $J^{\nu_{k}}=\mathbf{I}_{n_{x}+n_{z}}$ and $\sigma_{k} \neq \sigma_{k+1}$, then a switch occurs at time $t_{k}$ but no impulse.

For $i \in \mathcal{I}, j \in \mathcal{J}$, let

$$
A^{i}=\left(\begin{array}{cc}
A_{11}^{i} & A_{12}^{i} \\
A_{21}^{i} & A_{22}^{i}
\end{array}\right), J^{j}=\left(\begin{array}{cc}
J_{11}^{j} & J_{12}^{j} \\
J_{21}^{j} & J_{22}^{j}
\end{array}\right),
$$

where $A_{11}^{i}, J_{11}^{j} \in \mathbb{R}^{n_{x} \times n_{x}}$, and $A_{22}^{i}, A_{12}^{i}, A_{21}^{i}, J_{22}^{j}, J_{12}^{j}, J_{21}^{j}$ are of appropriate dimensions.

Let us impose the following standard assumption [5] in the singular perturbation theory framework:

Assumption 2: $A_{22}^{i}$ is non-singular for all $i \in \mathcal{I}$.

Then, we perform the following time dependent change of variable:

$$
\left(\begin{array}{l}
x(t) \\
y(t)
\end{array}\right)=P_{\sigma_{k}}\left(\begin{array}{c}
x(t) \\
z(t)
\end{array}\right), \forall t \in\left[t_{k}, t_{k+1}\right), k \in \mathbb{N}
$$

where, for all $i \in \mathcal{I}$

$$
P_{i}=\left(\begin{array}{cc}
\mathbf{I}_{n_{x}} & \mathbf{0}_{n_{x}, n_{z}} \\
\left(A_{22}^{i}\right)^{-1} A_{21}^{i} & \mathbf{I}_{n_{z}}
\end{array}\right) .
$$

It is worth noting that the matrix $P_{i}$ is invertible and for all $i \in \mathcal{I}$

$$
P_{i}^{-1}=\left(\begin{array}{cc}
\mathbf{I}_{n_{x}} & \mathbf{0}_{n_{x}, n_{z}} \\
-\left(A_{22}^{i}\right)^{-1} A_{21}^{i} & \mathbf{I}_{n_{z}}
\end{array}\right) .
$$


Using (6), the continuous dynamics (4) in the variables $x, y$ becomes:

$$
\begin{array}{r}
\left(\begin{array}{c}
\dot{x}(t) \\
\varepsilon \dot{y}(t)
\end{array}\right)=\left(\begin{array}{cc}
A_{0}^{\sigma_{k}} & B_{1}^{\sigma_{k}} \\
\varepsilon B_{2}^{\sigma_{k}} & A_{22}^{\sigma_{k}}+\varepsilon B_{3}^{\sigma_{k}}
\end{array}\right)\left(\begin{array}{c}
x(t) \\
y(t)
\end{array}\right) \\
\forall t \in\left(t_{k}, t_{k+1}\right], k \in \mathbb{N}
\end{array}
$$

where for all $i \in \mathcal{I}$ one has

$$
\begin{aligned}
& A_{0}^{i}=A_{11}^{i}-A_{12}^{i}\left(A_{22}^{i}\right)^{-1} A_{21}^{i}, B_{1}^{i}=A_{12}^{i}, \\
& B_{2}^{i}=\left(A_{22}^{i}\right)^{-1} A_{21}^{i} A_{0}^{i}, B_{3}^{i}=\left(A_{22}^{i}\right)^{-1} A_{21}^{i} A_{12}^{i} .
\end{aligned}
$$

Similarly, the jump map (5) is rewritten in the $x, y$ variables as:

$$
\left(\begin{array}{l}
x\left(t_{k}^{+}\right) \\
y\left(t_{k}^{+}\right)
\end{array}\right)=R_{\sigma_{k} \rightarrow \sigma_{k+1}}^{\nu_{k}}\left(\begin{array}{c}
x\left(t_{k}\right) \\
y\left(t_{k}\right)
\end{array}\right), \quad \forall k \geq 1
$$

where for all $i, i^{\prime} \in \mathcal{I}, j \in \mathcal{J}$,

$$
R_{i \rightarrow i^{\prime}}^{j}=P_{i^{\prime}} J^{j} P_{i}^{-1}
$$

Remark 2: It is worth emphasizing that the jump map $R_{\sigma_{k} \rightarrow \sigma_{k+1}}^{\nu_{k}}$ is uniquely defined i.e., when a jump and a switch occur simultaneously we can either consider that the switch precedes the jump or the reverse.

\section{Stability ANALYSis}

We study the stability of system (7)-(8) by using Lyapunov function arguments. We state the following result which is a particular case of [15, Theorem 1].

Proposition 4: Consider the hybrid system (7)-(8) and a positive scalar $\varepsilon_{\max }$. The equilibrium point $\left(x^{*}, y^{*}\right)=(0,0)$ is globally asymptotically stable, for all $\varepsilon \in\left[0, \varepsilon_{\max }\right)$ if there exists a function $t \mapsto V(x(t), y(t))$ such that $V(0,0)=0$, $V(x, y>0, \forall(x, y) \neq(0,0)$ and the following hold for any $(x(0), y(0)) \neq(0,0)$ :

(i) for all $\varepsilon \in\left[0, \varepsilon_{\max }\right)$ and $t \in\left(t_{k}, t_{k+1}\right]$

$$
\dot{V}(x(t), y(t))<0 \text {, }
$$

(ii) for all $t_{k}, k \in \mathbb{N}$

$$
V\left(x\left(t_{k+1}\right), y\left(t_{k+1}\right)\right)<V\left(x\left(t_{k}\right), y\left(t_{k}\right)\right)
$$

The stability analysis will be performed by considering the Lyapunov function defined by

$$
V(x, y)=x^{\top} Q_{s}^{\sigma_{k}} x+y^{\top} Q_{f}^{\sigma_{k}} y,
$$

and showing that under appropriate conditions $V$ satisfies Proposition 4. In the rest of the paper, we assume the stability of each mode of the slow and fast dynamics. In other words the following holds.

Assumption 3: $A_{0}^{i}$ and $A_{22}^{i}$ are Hurwitz for all $i \in \mathcal{I}$.

From the previous assumption, we can deduce that there exist symmetric positive definite matrices $Q_{s}^{i} \geq \mathbf{I}_{n_{x}}, Q_{f}^{i} \geq \mathbf{I}_{n_{z}}$, for $i \in \mathcal{I}$ and a positive number $\lambda$ such that

$$
\begin{array}{r}
A_{0}^{i^{\top}} Q_{s}^{i}+Q_{s}^{i} A_{0}^{i} \leq-2 \lambda Q_{s}^{i} \\
A_{22}^{i^{\top}} Q_{f}^{i}+Q_{f}^{i} A_{22}^{i} \leq-2 \lambda Q_{f}^{i}
\end{array}
$$

Remark 3: Proposition 1 states that there exists $\varepsilon_{1}>0$ such that the linear dynamics (4) are all stable for all $\varepsilon \in\left(0, \varepsilon_{1}\right)$.

In the sequel, the Lyapunov functions of the decoupled dynamics defined in section II become $W_{s}(t)=$ $\sqrt{x(t)^{\top} Q_{s}^{\sigma_{k}} x(t)}$ and $W_{f}(t)=\sqrt{y(t)^{\top} Q_{f}^{\sigma_{k}} y(t)}$ where $\sigma_{k} \in$ $\mathcal{I}$ is the corresponding mode over the time interval $\left(t_{k}, t_{k+1}\right]$. For each mode $i \in \mathcal{I}$, let $b_{1}^{i}=\left\|Q_{s}^{i} B_{1}^{i}\right\|, b_{2}^{i}=\left\|Q_{f}^{i} B_{2}^{i}\right\|$, $b_{3}^{i}=\left\|Q_{f}^{i} B_{3}^{i}\right\|$. Let $b_{h}=\max _{i \in \mathcal{I}} b_{h}^{i}, h \in\{1,2,3\}$ and define

$$
\beta_{1}=\frac{\max \left(b_{2}, b_{3}\right)}{\lambda\left(1-\varepsilon_{1}(1-\theta)\right)}, \beta_{2}=\frac{b_{1}}{\lambda\left(1-\varepsilon_{2}\right)}, \beta_{3}=\frac{b_{1} \beta_{1}}{\lambda \theta} .
$$

The next result characterizes the variation of $W_{s}$ and $W_{f}$ during continuous dynamics between two events.

Lemma 5: Under Assumption 3, let $\varepsilon \in\left(0, \varepsilon_{2}\right)$, and let $\tau_{k}=$ $t_{k+1}-t_{k}$ for a sequence $\left(t_{k}\right)_{k \geq 0}$ of event times. Then for all $k \in \mathbb{N}$,

$$
\begin{aligned}
W_{s}\left(t_{k+1}\right) & \leq W_{s}\left(t_{k}^{+}\right)\left(1+\varepsilon \beta_{3}\right) \mathrm{e}^{-\lambda(1-\theta) \tau_{k}} \\
& +W_{f}\left(t_{k}^{+}\right) \varepsilon\left(\beta_{2}+\beta_{3}\right) \mathrm{e}^{-\lambda(1-\theta) \tau_{k}} \\
W_{f}\left(t_{k+1}\right) & \leq W_{s}\left(t_{k}^{+}\right) \varepsilon \beta_{1} \mathrm{e}^{-\lambda(1-\theta) \tau_{k}} \\
& +W_{f}\left(t_{k}^{+}\right)\left(\mathrm{e}^{-\frac{\lambda}{\varepsilon} \tau_{k}}+\varepsilon \beta_{1} \mathrm{e}^{-\lambda(1-\theta) \tau_{k}}\right) .
\end{aligned}
$$

Proof: This is straightforward from Propositions 2 and 3 by remarking that $\sqrt{V} \leq W_{s}+W_{f}$ and $\mathrm{e}^{-\lambda t} \leq \mathrm{e}^{-(1-\theta) \lambda t}$.

In the following we complete the characterization of the variation of $W_{s}$ and $W_{f}$ by analyzing their behavior when an event occurs.

Remark 4: There exist a positive scalar $\alpha \geq 1$ such that, for all $i, i^{\prime} \in \mathcal{I}$

$$
\left(\begin{array}{cc}
Q_{s}^{i} & \mathbf{0}_{n_{x}, n_{z}} \\
\mathbf{0}_{n_{z}, n_{x}} & Q_{f}^{i}
\end{array}\right) \leq \alpha^{2}\left(\begin{array}{cc}
Q_{s}^{i^{\prime}} & \mathbf{0}_{n_{x}, n_{z}} \\
\mathbf{0}_{n_{z}, n_{x}} & Q_{f}^{i^{\prime}}
\end{array}\right)
$$

and let $\gamma \geq 0$ such that

$$
\gamma^{2}=\max _{i, i^{\prime} \in \mathcal{I}, j \in \mathcal{J}}\left\|\left(R_{i \rightarrow i^{\prime}}^{j}\right)^{\top}\left(\begin{array}{cc}
Q_{s}^{i} & \mathbf{0}_{n_{x}, n_{z}} \\
\mathbf{0}_{n_{z}, n_{x}} & Q_{f}^{i}
\end{array}\right) R_{i \rightarrow i^{\prime}}^{j}\right\|
$$

Remark 5: In order to keep the notation simple, we introduce the matrix :

$M_{\tau}=\left(\begin{array}{cc}\left(1+\varepsilon \beta_{3}\right) \mathrm{e}^{-\lambda(1-\theta) \tau_{k}} & \varepsilon\left(\beta_{2}+\beta_{3}\right) \mathrm{e}^{-\lambda(1-\theta) \tau_{k}} \\ \varepsilon \beta_{1} \mathrm{e}^{-\lambda(1-\theta) \tau_{k}} & \mathrm{e}^{-\frac{\lambda}{\varepsilon} \tau_{k}}+\varepsilon \beta_{1} \mathrm{e}^{-\lambda(1-\theta) \tau_{k}}\end{array}\right)$

Lemma 6: Let $\varepsilon_{1}$ as in Remark 3, $0<\varepsilon_{2}<\min \left(\varepsilon_{1}, 1\right)$ and let $\beta_{1}, \beta_{2}, \beta_{3}$ be defined by (9). Let $\alpha$ and $\gamma$ satisfying (12) and (13) respectively and let $\tau_{k}=t_{k+1}-t_{k}$ for a given sequence $\left(t_{k}\right)_{k \geq 0}$ of event times. Then, for all $k \in \mathbb{R}_{+}$,

$$
V\left(t_{k+1}\right) \leq \zeta^{2} \rho\left(M_{\tau}\right)^{2} V\left(t_{k}\right)
$$

where $\zeta=\sqrt{2} \alpha \gamma$ and $\rho\left(M_{\tau}\right)$ is the infinity norm of $M_{\tau}$ i.e. maximum absolute row sum of the matrix. 
Proof: Lemma 5 and Remark 4 yield

$$
\left(\begin{array}{l}
W_{s}\left(t_{k+1}\right) \\
W_{f}\left(t_{k+1}\right)
\end{array}\right) \leq M_{\tau}\left(\begin{array}{c}
W_{s}\left(t_{k}^{+}\right) \\
W_{f}\left(t_{k}^{+}\right)
\end{array}\right)
$$

where the inequality is meant component-wise. Consequently, using that $V(t)=\left\|\left(W_{s}(t), W_{f}(t)\right)^{\top}\right\|^{2}$ one obtains that

$$
\begin{aligned}
V\left(t_{k+1}\right) & =W_{s}\left(t_{k+1}\right)^{2}+W_{f}\left(t_{k+1}\right)^{2} \\
& \leq \rho\left(M_{\tau}\right)^{2}\left(W_{s}\left(t_{k}\right)+W_{f}\left(t_{k}\right)\right)^{2} \\
& \leq 2 \rho\left(M_{\tau}\right)^{2} V\left(t_{k}^{+}\right)
\end{aligned}
$$

On the other hand

$$
\begin{gathered}
V\left(t_{k}^{+}\right)=\left(\begin{array}{l}
x\left(t_{k}^{+}\right) \\
y\left(t_{k}^{+}\right)
\end{array}\right)^{\top}\left(\begin{array}{ll}
Q_{s}^{\sigma_{k+1}} & \mathbf{0}_{n_{x}, n_{z}} \\
\mathbf{0}_{n_{z}, n_{x}} & Q_{f}^{\sigma_{k+1}}
\end{array}\right)\left(\begin{array}{c}
x\left(t_{k}^{+}\right) \\
y\left(t_{k}^{+}\right)
\end{array}\right) \\
\leq\left(\begin{array}{c}
x\left(t_{k}\right) \\
y\left(t_{k}\right)
\end{array}\right)^{\top}\left(R_{\sigma_{k} \rightarrow \sigma_{k+1}}^{\nu_{k}}\right)^{\top}\left(\begin{array}{cc}
Q_{s}^{\sigma_{k+1}} & \mathbf{0}_{n_{x}, n_{z}} \\
\mathbf{0}_{n_{z}, n_{x}} & Q_{f}^{\sigma_{k+1}}
\end{array}\right) \\
R_{\sigma_{k} \rightarrow \sigma_{k+1}}^{\nu_{k}}\left(\begin{array}{c}
x\left(t_{k}\right) \\
y\left(t_{k}\right)
\end{array}\right) \\
\leq \alpha^{2}\left(\begin{array}{c}
x\left(t_{k}\right) \\
y\left(t_{k}\right)
\end{array}\right)^{\top}\left(R_{\sigma_{k} \rightarrow \sigma_{k+1}}^{\nu_{k}}\right)^{\top}\left(\begin{array}{cc}
Q_{s}^{\sigma_{k}} & \mathbf{0}_{n_{x}, n_{z}} \\
\mathbf{0}_{n_{z}, n_{x}} & Q_{f}^{\sigma_{k}}
\end{array}\right) \\
R_{\sigma_{k} \rightarrow \sigma_{k+1}}^{\nu_{k}}\left(\begin{array}{l}
x\left(t_{k}\right) \\
y\left(t_{k}\right)
\end{array}\right) \\
\leq \alpha^{2} \gamma^{2}\left\|\left(\begin{array}{l}
x\left(t_{k}\right) \\
y\left(t_{k}\right)
\end{array}\right)\right\|^{2}
\end{gathered}
$$

Using that, for all $i \in \mathcal{I}$ one has $Q_{s}^{i} \geq \mathbf{I}_{n_{x}}$ and $Q_{f}^{i} \geq \mathbf{I}_{n_{z}}$, it follows that

$$
\left\|\left(\begin{array}{l}
x\left(t_{k}\right) \\
y\left(t_{k}\right)
\end{array}\right)\right\|^{2} \leq V\left(t_{k}\right)
$$

yielding that (14) holds.

We can now state the main result of this paper.

Theorem 7: Consider the singularly perturbed system (4)(5). Suppose that Assumptions 3 hold and $\varepsilon_{1}$ such that $0<\varepsilon_{1}<\frac{1}{\frac{\left(b_{1}+b_{2}\right)^{2}}{4 \theta \lambda^{2}}+\frac{b_{3}}{\lambda}+1-\theta}$. Let also consider $\alpha$ and $\gamma$ such that (12) and (13) hold. Let $0<\varepsilon_{2}<\min \left(\varepsilon_{1}, 1\right)$ and let $0<\varepsilon_{3}<\min \left(\varepsilon_{2}, \frac{1}{\beta_{2}+2 \beta_{3}}, \frac{1}{2 \zeta \beta_{1}}\right)$.

Then, for all $\varepsilon \in\left(0, \varepsilon_{3}\right)$, the system is globally asymptotically stable if the inter-event time $\tau_{k}$ satisfies :

$$
\begin{aligned}
\tau_{k}>\frac{1}{\lambda} \max & \left\{\frac { 1 } { ( 1 - \theta ) } \left(\ln (\zeta)+\varepsilon\left(\beta_{2}+2 \beta_{3}\right),\right.\right. \\
\varepsilon & \left.\ln \left(\frac{\zeta}{1-2 \zeta \varepsilon \beta_{1}}\right)\right\}
\end{aligned}
$$

where $\zeta=\alpha \gamma$ and $\beta_{1}, \beta_{2}, \beta_{3}$ are given in Propositions 2-3.

Proof: In view of Assumptions 3 item (i) of Proposition 4 holds for all $\varepsilon \in\left(0, \varepsilon_{1}\right)$. To prove that inter-event time $\tau_{k}$ given by (15) guarantees the stability of the singularly perturbed system given by (4)-(5), we analyze the behavior of the Lyapunov function and prove that:

$$
V\left(t_{k+1}\right)<V\left(t_{k}\right), \quad \forall k \in \mathbb{R}^{+} .
$$

From Lemma 6, this is the case if all row sums of $\zeta M_{\tau}$ are strictly smaller than 1 . In other words, the system is globally asymptotically stable if the following inequalities hold

$$
\begin{aligned}
\zeta\left(1+\varepsilon\left(\beta_{2}+2 \beta_{3}\right)\right) \mathrm{e}^{-\lambda(1-\theta) \tau_{k}} & <1 \\
\zeta\left(2 \varepsilon \beta_{1} \mathrm{e}^{-\lambda(1-\theta) \tau_{k}}+\mathrm{e}^{-\frac{\lambda}{\varepsilon} \tau_{k}}\right) & <1
\end{aligned}
$$

Since $\mathrm{e}^{-(1-\theta) \lambda \tau_{k}} \leq 1$ for all $\theta \in(0,1)$, it follows :

$$
\begin{aligned}
\mathrm{e}^{-\lambda(1-\theta) \tau_{k}}\left(1+\varepsilon\left(\beta_{2}+2 \beta_{3}\right)\right) & <\frac{1}{\zeta} \\
2 \varepsilon \beta_{1}+\mathrm{e}^{-\frac{\lambda}{\varepsilon} \tau_{k}} & <\frac{1}{\zeta}
\end{aligned}
$$

These are equivalent to

$$
\begin{aligned}
\tau_{k} & >\frac{1}{\lambda(1-\theta)} \ln \left(\zeta\left(1+\varepsilon\left(\beta_{2}+2 \beta_{3}\right)\right)\right) \\
\tau_{k} & >\frac{\varepsilon}{\lambda} \ln \left(\frac{\zeta}{1-2 \zeta \varepsilon \beta_{1}}\right)
\end{aligned}
$$

Using that $\ln \left(1+\varepsilon\left(\beta_{2}+2 \beta_{3}\right)\right) \leq \varepsilon\left(\beta_{2}+2 \beta_{3}\right)$ for all $\varepsilon \geq 0$, one gets that (16), (17) hold as far as:

$$
\begin{aligned}
\tau_{k} & >\frac{1}{\lambda(1-\theta)}\left(\ln (\zeta)+\varepsilon\left(\beta_{2}+2 \beta_{3}\right)\right) \\
\tau_{k} & >\frac{\varepsilon}{\lambda} \ln \left(\frac{\zeta}{1-2 \zeta \varepsilon \beta_{1}}\right)
\end{aligned}
$$

These inequalities are satisfied if (15) holds.

Remark 6: Let

$$
\begin{aligned}
\tau^{*}=\frac{1}{\lambda} \max & \left\{\frac { 1 } { ( 1 - \theta ) } \left(\ln (\zeta)+\varepsilon\left(\beta_{2}+2 \beta_{3}\right),\right.\right. \\
& \left.\varepsilon \ln \left(\frac{\zeta}{1-2 \zeta \varepsilon \beta_{1}}\right)\right\} .
\end{aligned}
$$

Theorem 7 gives a lower bound on the dwell time between two consequent events ensuring the two time scale system (4)-(5) is globally asymptotically stable. However, this lower bound on the dwell time condition may be subject to some conservatism. This means that the overall system can be stable when the time interval between consecutive events is less than $\tau^{*}$.

A closer look at $\tau^{*}$ reveals that:

$$
\tau^{*}=\max (\mathcal{O}(1)+\mathcal{O}(\varepsilon), \mathcal{O}(\varepsilon))
$$

In Corollary 1 we show that $\mathcal{O}(1)$ term vanishes when the stability of the slow dynamics is not affected by the discrete events. On the other hand we also show that the $\mathcal{O}(1)$ term is required for stability of linear impulsive and/or switched systems evolving on only one time-scale (see Proposition 8).

Corollary 1: Let us suppose that all the modes of the slow dynamics with jumps in (4) - (5) share a common Lyapunov function. Thus (12) can be reformulated as: there exist positive scalars $\gamma_{1}, \gamma_{2}>0$ such that

$$
W_{f}\left(t_{k}^{+}\right) \leq \gamma_{1} W_{s}\left(t_{k}\right)+\gamma_{2} W_{f}\left(t_{k}\right) \text { and } W_{s}\left(t_{k}^{+}\right) \leq W_{s}\left(t_{k}\right)
$$


Consequently, the minimum dwell time in (15) guaranteeing the global asymptotic stability becomes

$$
\begin{aligned}
\tau^{*}=\frac{\varepsilon}{\lambda} \max & \left\{\beta_{3}+\left(\beta_{2}+2 \beta_{3}\right)\left(\gamma_{1}+\gamma_{2}\right),\right. \\
& \left.\ln \left(\frac{\gamma_{1}+\gamma_{2}}{1-\gamma \varepsilon \beta_{1}\left(1+\gamma_{1}+\gamma_{2}\right)}\right)\right\}=\mathcal{O}(\varepsilon) .
\end{aligned}
$$

Remark 7: - In Corollary 1 the dwell time is closely related to the singular perturbation parameter $\varepsilon$. The length of the time interval between two consecutive events is lower bounded by the time it takes for fast dynamics to compensate the potential increase of Lyapunov function at jumps or/and switchings.

- When $\mathcal{J}=\emptyset$, Corollary 1 improves the result [11], [3] by emphasizing that the dwell time is $\mathcal{O}(\varepsilon)$ if all the slow dynamics share a common Lyapunov function.

Let us now study the meaning of $\mathcal{O}(1)$ term in the expression of $\tau^{*}$. To do that, we consider the following linear impulsive switched system

$$
\left\{\begin{aligned}
\dot{x}(t) & =A^{\sigma_{k}} x(t) \quad \forall t \in\left(t_{k}, t_{k+1}\right] \\
x\left(t_{k}^{+}\right) & =J^{\nu_{k}} x\left(t_{k}\right)
\end{aligned}\right.
$$

where the state $x(t)$ belongs to $\mathbb{R}^{n_{x}}$.

Let us assume that the matrices $A^{i}$ are all Hurwitz. We note again that there exist symmetric definite positive matrices $Q^{i} \geq \mathbf{I}_{n_{x}}$ and $\lambda \in \mathbb{R}_{+}$such that

$$
\left(A^{i}\right)^{\top} Q^{i}+Q^{i} A^{i} \leq-2 \lambda Q^{i}
$$

Let $\widehat{\alpha} \geq 1$ such that for all $i, j \in \mathcal{I}$ one has

$$
Q^{i} \leq \widehat{\alpha}^{2} Q^{j}
$$

and define $\widehat{\zeta}=\widehat{\alpha} \sqrt{\max _{i \in \mathcal{I}, j \in \mathcal{J}\left\|\left(J^{j}\right)^{\top} Q^{i} J^{j}\right\|}}$.

Remark 8: Notice that parameters $\widehat{\alpha}$ and $\widehat{\zeta}$ are equivalent to $\alpha, \zeta$ respectively in the two-time scale case.

Proposition 8: System (19) is asymptotically stable if the following inequality holds

$$
t_{k+1}-t_{k} \geq \frac{\ln (\widehat{\zeta})}{\lambda}
$$

\section{ILLUSTRATIVE EXAMPLES}

In this section, three examples of hybrid systems are used to verify the performance of the proposed strategy. In the first example, we assume that discontinuities only occur due to the switching signals. The second example is given to demonstrate the validity of the results above for impulsive systems. In the last example, we suppose that both jumps and switchings may occur at the same time.

\section{A. Switched system}

Let us introduce the singularly perturbed system (4)-(5) with $J^{\nu_{k}}=\mathbf{I}_{n_{x}+n_{z}}$ and $\sigma_{k} \in \mathcal{I}=\{1,2\}$. Let us consider a switched system having two modes defined by:

$$
\begin{aligned}
& A_{11}^{1}=-1, A_{12}^{1}=5, A_{21}^{1}=0, A_{22}^{1}=-1 . \\
& A_{11}^{2}=-1, A_{12}^{2}=0, A_{21}^{2}=5, A_{22}^{2}=-1 .
\end{aligned}
$$

Straightforwardly one has $A_{0}^{\sigma}=-1$ and $A_{22}^{\sigma}=-1$ i.e., the reduced (slow) system and the boundary layer (fast) system are stable. Nevertheless, as shown in [12], for any $\varepsilon>0$ there exists a periodic switching signal that renders the overall system unstable. To illustrate this, we fix $\varepsilon=0.003$ and let the initial condition $(x(0), z(0))^{\top}=(1,1)^{\top}$. It can be seen in Figures 1 and 2 that the switched singularly perturbed system is unstable for a periodic switching signal $\sigma$ of period $0.02 \mathrm{sec}$.

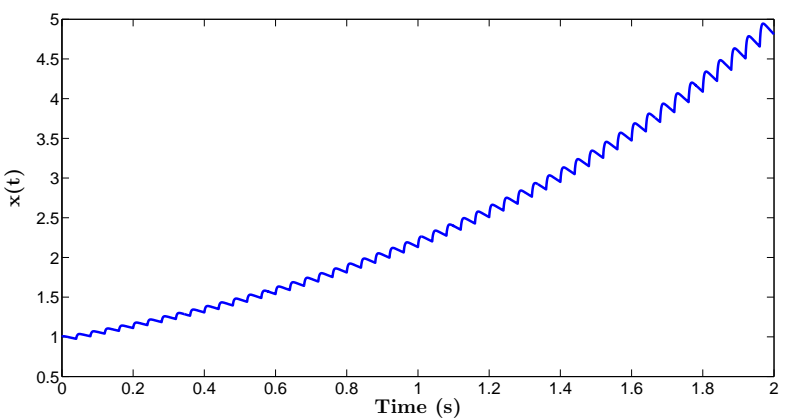

Fig. 1. State trajectories with $\tau_{k}=0.02 \mathrm{sec}$

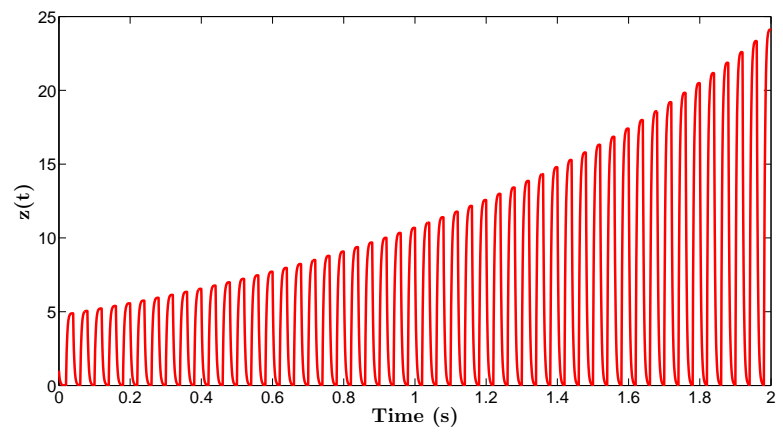

Fig. 2. State trajectories with $\tau_{k}=0.02 \mathrm{sec}$

It is noteworthy that, for $\varepsilon_{3}=0.0048$ and $\varepsilon=0.003$, the dwell-time condition in Corollary 1 yields $\tau_{k}=0.023 \mathrm{sec}$. Indeed considering a dwell-time larger than $0.023 \mathrm{sec}$ the system becomes stable.

\section{B. Reset system}

Consider now the singularly perturbed system (4)-(5) with a continuous dynamics defined by the matrix $A^{2}$ in (22). The reset map is described by a single matrix:

$$
J=\left(\begin{array}{ll}
0.48 & 0.49 \\
0.39 & 0.59
\end{array}\right)
$$


As in the previous example, the matrices $A_{0}$ and $A_{22}$ are Hurwitz, i.e. Assumptions 3 hold. Choosing the same value of $\varepsilon$ and starting with the same initial condition as in the previous example, the obtained value of the required interevent period stabilizing the overall system is given by $\tau_{k}=$ $1.255 \mathrm{sec}$. Simulating the dynamics with periodic jumps of period $1.2 \mathrm{sec}$ we noticed that the trajectories diverge. On the other hand, the simulation result in Figure 3 shows that the period of $1.255 \mathrm{sec}$ between two consecutive resets is sufficiently large and the continuous dynamics last enough to compensate the increase of states during jumps, which render the system asymptotically stable.

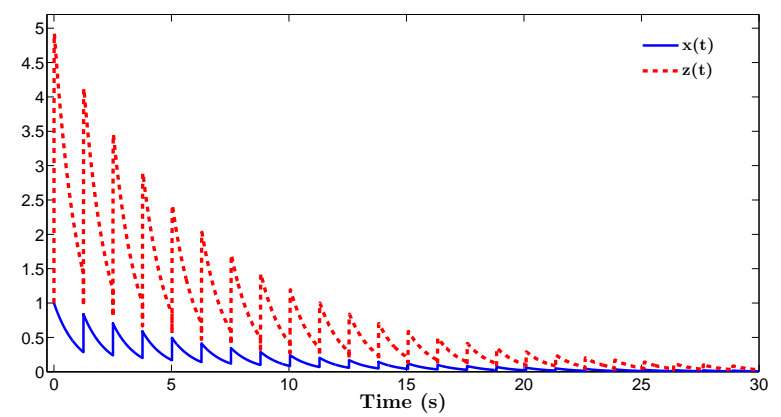

Fig. 3. State trajectories with $\tau_{k}=1.255 \mathrm{sec}$

\section{Hybrid system}

Let us consider the system (4)-(5) with matrices (21)-(23). Setting again $\varepsilon=0.003$ and applying Theorem 7 we obtain a period separating two consecutive events that has to be larger than $\tau_{k}=1.95 \mathrm{sec}$. In this case the system is asymptotically stable for all time periods lower-bounded by $\tau_{k}$. The trajectories of system (4)-(5) for $\tau_{k}=1.95 \mathrm{sec}$ are shown in Figure 4.

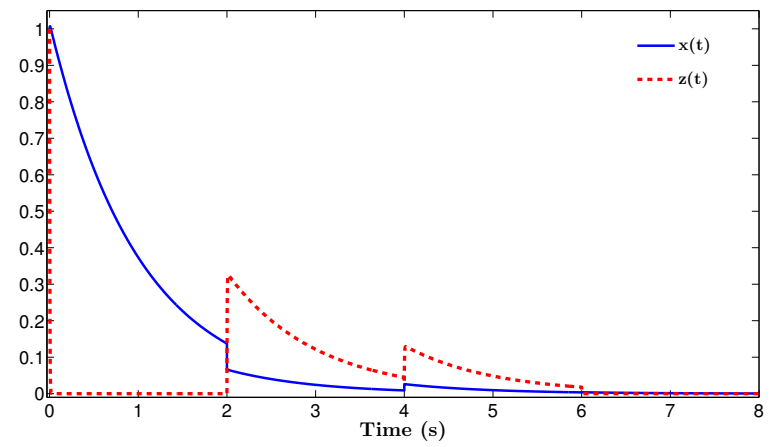

Fig. 4. State trajectories with $\tau_{k}=2 \mathrm{sec}$

Notice that $A_{21}^{1}=0$ explaining the very fast convergence of $z$ during the first period. At $t_{1}=2 \mathrm{~s}$, both a switch and a jump occur simultaneously. We also note that $A_{21}^{2} \neq 0$ meaning that the convergence speed of $z$ is slower due to the influence of the slow variable $x$. At time $t_{2}=2 t_{1}$ only a jump occurs meaning that the slow variable $x$ continues to affect the dynamics of $z$.

\section{CONCLUSIONS}

In this paper we proposed sufficient dwell-time condition for the stability of two-time scales switched impulsive systems. We emphasized that the required dwell-time consists of a sum of two terms. The first one corresponds to the stabilization of the slow dynamics while the second one is of order of the parameter characterizing the time-scale separation and is required for the stabilization of the dynamics on the slow manifold. The results have been illustrated by numerical simulation of linear systems affected by switches and/or state jumps.

\section{REFERENCES}

[1] L.Chen and K. Aihara, "A model of periodic oscillation for genetic regulatory systems," IEEE Transactions on Circuits and Systems, vol. 49, no. 10, pp. 1429-1436, 2002.

[2] A. Hodgkin and A. Huxley, "A quantitative description of membrane current and its application to conduction and excitation in nerve," The Journal of Physiology, vol. 117, pp. 500-544, 1952.

[3] I. Malloci, "Two time scale switched systems: Application to steering control in hot strip mills," Ph.D. dissertation, Univ. Lorraine - CRAN UMR 7039, 2009.

[4] R. Sanfelice and A. R. Teel, "On singular perturbations due to fast actuators in hybrid control systems," Automatica, vol. 47, pp. 692-701, 2011.

[5] P. Kokotović, H. Khalil, and J. O'Reilly, Singular perturbation methods in control: analysis and design. SIAM Series in Classics and Applied Mathematics, 1999.

[6] H. Khalil, Nonlinear Systems (Third Edition). Prentice Hall, 2001.

[7] M. Balachandra and P. R. Sethna, "A generalization of the method of averaging for systems with two time scales," Archive for Rational Mechanics and Analysis, vol. 58, no. 3, pp. 261-283, 1975.

[8] D. Nesic and A. Teel, "Input-to-state stability for nonlinear timevarying systems via averaging," Mathematics of Control, Signals, and Systems, vol. 14, pp. 257-280, 2001.

[9] A. Teel, L. Moreau, and D. Nesic, "A unified framework for input-tostate stability in systems with two time scales," IEEE Transactions on Automatic Control, vol. 48, no. 9, pp. 1526-1544, 2003.

[10] G. Hek, "Geometric singular perturbation theory in biological practice," Journal of Mathematical Biology, vol. 60, pp. 347-386, 2010.

[11] M.Alwan, X. Liu, and B. Ingalls, "Exponential stability of singularly perturbed switched systems with time delay," Nonlinear Analysis: Hybrid Systems, vol. 2, no. 3, pp. 913-921, 2008.

[12] I. Malloci, J. Daafouz, and C. Iung, "Stabilization of continuous-time singularly perturbed switched systems," in Proc. of the 48th IEEE Conference on Decision and Control, 2009.

[13] M. Abdelrahim, R. Postoyan, and J. Daafouz, "Event-triggered control of nonlinear singularly perturbed systems based only on the slow dynamics," Automatica, vol. 52, pp. 15-22, 2015.

[14] R. Goebel, R. Sanfelice, and A. Teel, Hybrid Dynamical Systems. Princeton University Press, 2012.

[15] L. Hetel, J. Daafouz, S. Tarbouriech, and C. Prieur, "Stabilization of linear impulsive systems through a nearly-periodic reset," Nonlinear Analysis: Hybrid Systems, vol. 7, pp. 4-15, 2013. 